\title{
ECONOMICAL EFFECTS OF ON-LINE ELEMENTAL ANALYSIS PERFORMANCE ON FLOTATION CONTROL
}

\author{
Remes A. ${ }^{1)}$, Kongas, M. ${ }^{2)}$, Saloheimo, K. ${ }^{2)}$, Jämsä-Jounela, S-L. ${ }^{1)}$ \\ 1) Helsinki University of Technology \\ Department of Chemical Technology \\ Laboratory of Process Control and Automation \\ P.O.Box 6100, FIN-02015 HUT, Finland \\ E-mail: Sirkka-l@hut.fi \\ 2) Outokumpu Technology, P.O.Box 84, FIN-02201 Espoo, Finland
}

\begin{abstract}
The paper studies the control performance of the flotation process as a function of the measurement accuracy and sampling frequency of an on-stream XRF analyzer, using a simple dynamic model. An additional aim is to assess how the economical result of a mineral flotation process depends on the quality of process control based on the online elemental assays. The economical performance of the control was studied by calculating the overall net smelter return with various analyzer parameter combinations. The economical performance of the simulated process declines strongly when the sampling cycle is increased. Copyright @ 2005 IFAC
\end{abstract}

Keywords: Flotation process control, Economical optimization, On-stream analysis, Xray fluorescence.

\section{INTRODUCTION}

Flotation control, either operator-based or a closed-loop advanced system, is dependent on the primary information from plant instrumentation, on-stream analyzers and laboratory. Obviously, the accuracy of the measurements translates directly into the accuracy of controlled variables in the process. Together with the variation in ore feed, and the dynamic behaviour of the multiple process stages and single unit processes, the timedependent nature of the measurements (measurement delay and sampling frequency) is also a significant factor. In this respect the assays used for process control have different quality requirements compared with the assays used for material balances based on composite samples.

Control of the flotation process requires concentration measurements at several process points. An XRF analyzer measures the concentrations of slurry lines in a continuous cycle. The control performance depends on the analytical accuracy and sampling frequency of the concentration measurement. With a long cycle time, the state of the process changes before a new measurement becomes available. The XRF technique is based on the measurement of X-ray fluorescence and scatter intensities. With short measurement times the statistical variance of the counted X-ray pulses increases, thereby increasing the random error in the analysis. In addition, the measurement accuracy depends on the concentration of the component to be measured in the slurry. The total error due to limited measurement accuracy and sampling frequency can be minimized by optimizing the combination of measurement time and cycle time for each of the measurement lines.

A few published studies deal with the importance of the accuracy and precision of the on-line analysis for flotation control. Cooper (1976) has investigated the measurement accuracies of in-stream and multiplexed on- 
stream approaches and estimated the return of the analyzer investment. Flintoff (1992) examined the relationship between on-stream analysis accuracy to controlled concentrate grade and recovery. Tenno et al. (1996) used Monte Carlo simulation of plant history data for estimating the effect of analysis error on the economics of a flotation process.

\section{SPEED AND ACCURACY OF ON-LINE ELEMENTAL ANALYSIS}

\subsection{On-line XRF analysis}

In X-ray fluorescence (XRF) analysis, the primary radiation from an excitation source (Xray tube or radioactive isotope) excites sample atoms, or knocks out inner shell electrons in the sample atoms (see Fig. 1). An electron from a higher shell fills the electron vacancy in order to keep the atom stable. The energy difference between the shells is emitted as an X-ray fluorescence photon. The energy of the emitted radiation photon is characteristic to each individual element in the periodic table. An XRF analyzer contains a detector or series of detectors to measure and convert the emitted radiation photons into characteristic energy intensities corresponding to specific elements, for example, copper $(\mathrm{Cu})$, nickel $(\mathrm{Ni})$ or zinc $(\mathrm{Zn})$. This intensity information is then converted to assays using a calibration model.

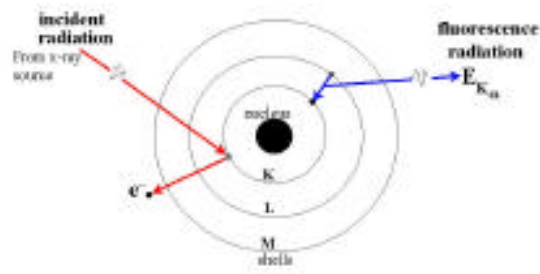

Fig. 1. Principle of X-ray fluorescence.

The fluorescence intensities can be measured using wavelength dispersive spectrometers (WDX) for each element and solids content, or energy dispersive (EDX) detectors that measure the whole fluorescence spectrum.

The assays from the analyzer indicate the percentage by weight of the concentration of an element out of the total solids. To compensate for variation in the solids content in the sample, the measured element specific intensities are corrected by measuring the backscatter of the primary radiation from the sample.

\subsection{Analysis speed and accuracy}

The analysis speed of an XRF analyzer is greatly dependent on how the measurement is configured. One of the analysis error components is due to the random variance of the counted intensities. This component is inversely proportional to the measurement time, i.e. the relative variance decreases when the number of counted pulses becomes larger. As a general rule, the more X-rays available in the primary radiation and which can be counted by the detector system, the faster the measurement can be made to achieve satisfactory accuracy. The modern on-stream system is capable of measuring up to 24 streams with a typical speed of $30 \mathrm{~s} / \mathrm{sample}$ line.

The accuracy of the analysis is dependent on how the slurry sample is taken and how it is presented to the analysis. The primary samplers are specifically designed to handle a particular process application and flow conditions. The sample representativeness depends on adequate mixing of the sample and isokinetic slurry flow to the sample line.

Because of X-ray absorption by water and solids, the measured fluorescence occurs in a thin (below $1 \mathrm{~mm}$ for most elements of interest) layer immediately in front of the plastic window separating the continuously flowing sample from the X-ray source and detector (See Fig. 2). Slurry flow in the measurement cell is of fundamental importance to the overall accuracy. The cell design must ensure that all particle sizes pass through the narrow region at the surface of the window. The window must remain clean and the sample in the sensitive volume must be representative and free from air. Sample flow against the window prevents scaling and continuously replenishes the layer with fresh representative sample.

The accuracy of XRF analysis is also affected by variations in particle size, sample composition, mineralization and slurry density. Depending on the application, the relative analysis accuracy can vary between $1-10 \%$. 


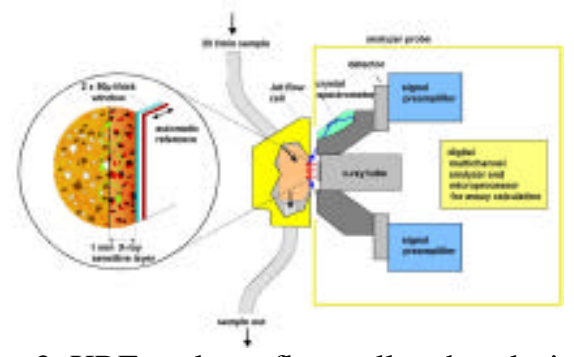

Fig. 2. XRF analyzer flow cell and analysis technology.

\section{DYNAMIC SIMULATION MODEL}

This study is focused on assessing the metallurgical performance of a flotation circuit as a function of the on-stream analysis system parameters. A model is therefore required for both the dynamics of the flotation process and the process control based on the on-stream assaying. Figure 3 shows a schematic diagram of the model used in the simulations. A simplification has been done to point out the major effects. The model was implemented in PC-Matlab/Simulink environment.

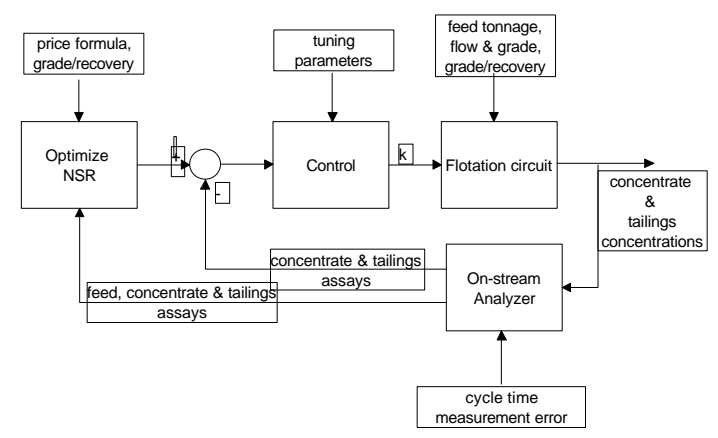

Fig. 3. The structure of the simulation model.

\subsection{Flotation circuit model}

Flotation circuit modeling has been studied by various authors, and a number of models have been proposed. In this model, the main objective is to study the behavior of a flotation circuit under tight control. Simplifications have been made in order to keep the number of experimental parameters reasonable. To model the metal concentrations in the main slurry streams we use the simplest classical first-order reaction kinetics equations, packing the flotation kinetics into a single parameter, the flotation rate constant.

Suppose we feed slurry with flow $F_{f}$ and metal concentration $\mathrm{C}_{\mathrm{f}}$ to a flotation stage with slurry volume V. Assuming ideal mixing in the slurry, the dynamic mass balance between the concentrate and tailings flows is written as (Jämsä-Jounela, 1990)

$\frac{d m}{d t}=F_{f} C_{f}-F_{t} C_{t}-k V C_{t}$,

from which the slurry and tailings concentration is obtained

$$
\frac{d C_{t}}{d t}=\frac{1}{V}\left(F_{f} C_{f}-F_{t} C_{t}\right)-k C_{t}
$$

where $F_{t}$ is the tailings flow $\left(\mathrm{dm}^{3} / \mathrm{min}\right), \mathrm{C}_{\mathrm{t}}$ is the metal volume concentration in the slurry $\left(\mathrm{kg} / \mathrm{dm}^{3}\right)$ and $\mathrm{k}$ is the flotation rate constant $(1 / \mathrm{min})$. The concentration of metals in the foam phase follows the first order reaction equation, if the operation is under free flotation conditions (Jämsä-Jounela, 1990). If the density of the slurry is high, the feed concentration is high, or the flotation air feed is low, then the process can be under inhibited flotation, in which the area of the air bubbles is a limiting factor. Another condition that is not covered by equation (2) is when the structure of the foam limits efficient flow to the launder (Lynch, et al., 1981).

In addition, the water balance equation is defined in order to solve for the volume flows. When the total concentrate flow is calculated the froth washing and launder water are also taken into account.

\subsection{Grade-recovery relationship}

Estimation of the grade-recovery dependency from process data is not a straightforward task. Typical data points of the feed (\%), concentrate $(\%)$ and recovery $(\%)$ values of an industrial copper flotation circuit are shown in figure 4 .

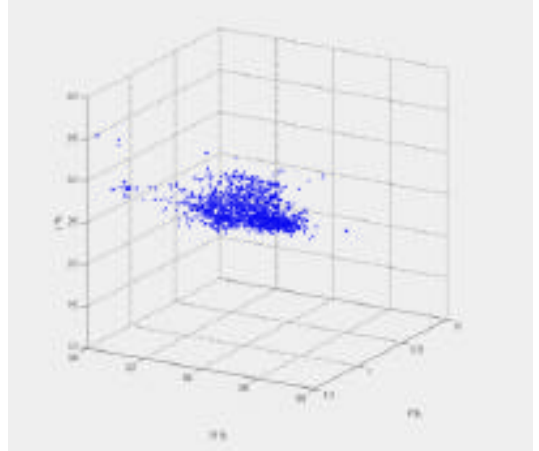

Fig. 4. Feed, concentrate, recovery -data from an industrial flotation circuit.

Several types of models, including second order surface, average grid and neural network 
models, were evaluated to fit the data shown in Fig. 4. However, the best results were achieved with an exponential curve fitting. Equation 3 was fitted to over three thousand data points by using the least squares method.

$$
R=R_{\max }\left\lfloor 1-e^{\left(\left(c-c_{\max }\right) / k\right)}\right\rfloor
$$

In equation (3) $\mathrm{R}(\%)$ is recovery, $\mathrm{R}_{\max }(\%)$ is a maximum recovery, $\mathrm{c}(\%)$ concentration, $\mathrm{c}_{\max }$ $(\%)$ is the maximum concentration and $\mathrm{k}$ is an estimated parameter. Equation (3) was applied separately to four data classes, in which the data points were classified into quartiles according to the feed concentration. To avoid spurious local minima, the following constraints were applied: $\mathrm{R}$ should monotonously decrease when $\mathrm{c}$ decreases and $\mathrm{R}$ should monotonously increase when feed $(\%)$ increases.

\subsection{On-stream analyzer and assays}

The on-stream analyzer dynamics was modeled by a zero-order-hold circuit with a time delay describing the length of the analysis cycle. Thus the assay value remains constant until a new update is obtained after the cycle time $t_{\text {cycle }}$ has passed.

The analysis accuracy was simulated by adding additional random error to the 'actual' concentrations obtained from the model. To assess the effect of the sample measurement time, the random error was divided into two parts: a constant analysis error and a dynamic part accounting for the statistical component of the X-ray pulse. The latter component is inversely proportional to the square root of the measurement time $t_{\text {meas }}$,

$$
\begin{gathered}
\sigma_{t}=\sqrt{\sigma_{t 0}{ }^{2}+\left(p^{2} / t_{\text {meas }}\right)}, j t_{\text {cycle }}<t<(j+1) t_{\text {cycle }}, \\
j=1,2,3, \ldots
\end{gathered}
$$

Thus the simulated assays $\tilde{M}$ are

$$
\begin{aligned}
& \tilde{M}_{T}(t)=M_{T}\left(j t_{\text {cycle }}\right)+e_{0}\left(0, \sigma_{t 0}\right)+e_{1}\left(0, \sigma_{t 1}\left(t_{\text {meas }}\right)\right), \\
& j t_{\text {cycle }}<t<(j+1) t_{\text {cycle }}, j=1,2,3, \ldots
\end{aligned}
$$

where $t_{c y c l e}=$ cycle time, $\theta$ is the concentrate assay delay and $e(m, \sigma)$ is a normally distributed random number with mean $m$ and standard deviation $\sigma$.
3.4 Net smelter return optimization and control of the model

The control system in the model is based on a PI loop that adjusts the flotation rate constant in eq. (2). The objective is to keep the simulated final concentrate grade or tailing concentration at an optimal set point. The optimal operating point is determined from the intersections of the graderecovery curves, as shown in an example case of copper flotation in Fig. 5, and the isoeconomic curves for each ore feed grade. The economic values, and subsequent isoeconomic curves, are calculated from a formula for net smelter return (Kongas et al., 2001),

$$
N S R=f R\left(C_{1}-C_{2}-C_{3} / c\right)
$$

where $\mathrm{f}$ is the feed concentration, $\mathrm{R}$ is the recovery, $C_{1}$ is the metal price, $C_{2}$ is the treatment charge, $\mathrm{C}_{3}$ is the transport cost losses, and $\mathrm{c}$ is the concentration of the concentrate.

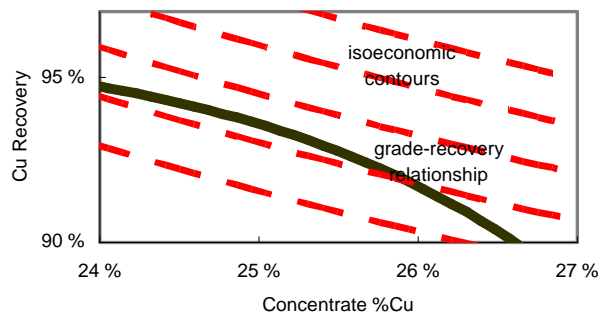

Fig. 5. Grade-recovery curves and isoeconomic contours of a flotation circuit.

\section{SIMULATED RESULTS OF THE ECONOMICAL PERFORMANCE OF THE FLOTATION PROCESS}

\subsection{Case description}

The case studied with the model was the copper flotation circuit at Inmet's Pyhäsalmi Mine in Central Finland. The process and the on-stream analyzer system have been described by Lähteenmäki et al. (1999). The open flotation circuit, well-maintained Courier ${ }^{\circledR}$ and advanced control, implemented in the Proscon $\AA$ automation system make the case ideal for these studies.

The data consist of a 3-day process history. The assays are recorded once per minute, and the analyzer cycle is 5-6 minutes. The feed tonnage and flow measurement data were also fed into the model. 
The residence time in the rougher/scavenger flotation cells is 24 minutes, as calculated by the feed volume flow rate and flotation cell volume (8 Outokumpu OK-16 flotation machines) and the first maximum in the cross-correlation between the feed and concentrate assays occurs with a delay of 20 minutes.

The fitted grade-recovery relationship at various feed grades is plotted in Figure 6. Even though the fit seems rather noisy it was used in the calculations to describe the average behaviour of the circuit.

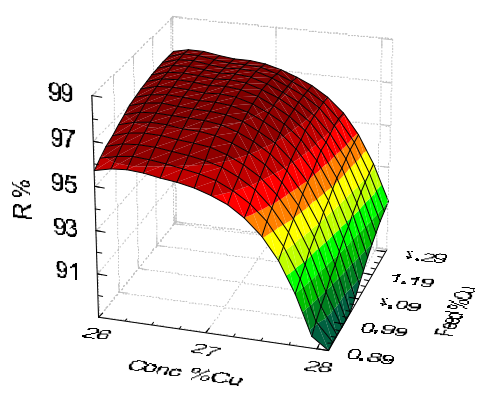

Fig. 5. The grade-recovery model at various feed grades.

Based on the calibration data, the analyzer system was described by the time-dependent error plotted in Figure 6. The simulation runs tried several combinations of measurement time and analysis cycle to cover the full range of reasonable values. Measurement times smaller than 10 seconds are impractical since the analyzer system requires a certain amount of time for sample changeover and flow stabilization.

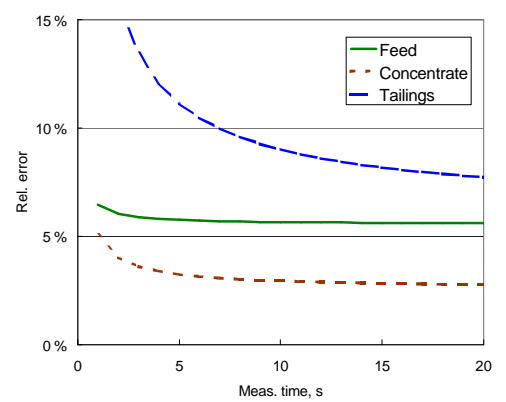

Fig. 6. The analyzer accuracy as a function of measurement time.

\subsection{Simulation runs with process data}

A total of 70 simulation runs with industrial flotation circuit data were performed. In each simulation the control interval (measurement cycle) and measurement accuracy (sample measurement time) were altered, and the net smelter return for the period was calculated. The simulation is based on three days' historical data from a copper flotation circuit. The NSR is scaled to correspond to $1.3 \mathrm{MTon} / \mathrm{yr}$ mining production; a typical price value in eq. (6) was used. Figure 7 shows the results of the simulations, at various sample measurement times, as a function of the analyzer cycle time. The fluctuations are due to the random nature of the simulations, the measurement error added as random numbers to the assay data.

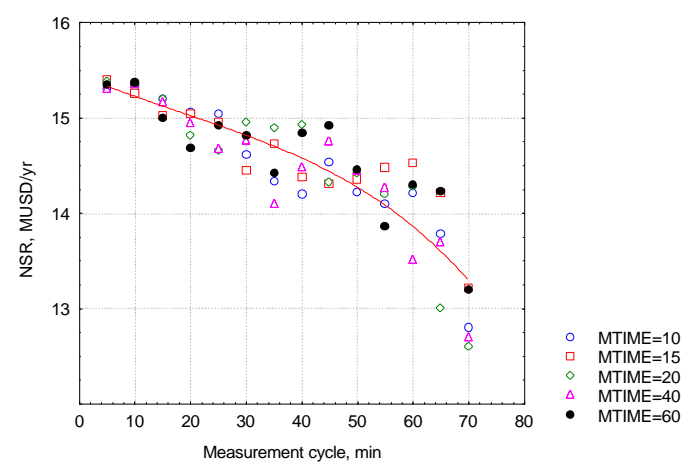

Fig. 7. Simulated economical results (NSR) as a function of measurement cycle with different measurement times.

Figure 8 shows a smoothed surface plot through the data. Based on the results, it is evident that the sample measurement time does not have a significant effect on the economical results. Instead, if the control actions are entirely based on assays and the measurement cycle becomes relatively long, then the measurement cycle has a significant effect in terms of economical value of the production. In this case the difference between an immediate measurement and a 40minute cycle is 1 MUSD/year, lost in nonoptimal and poor control of the flotation circuit.

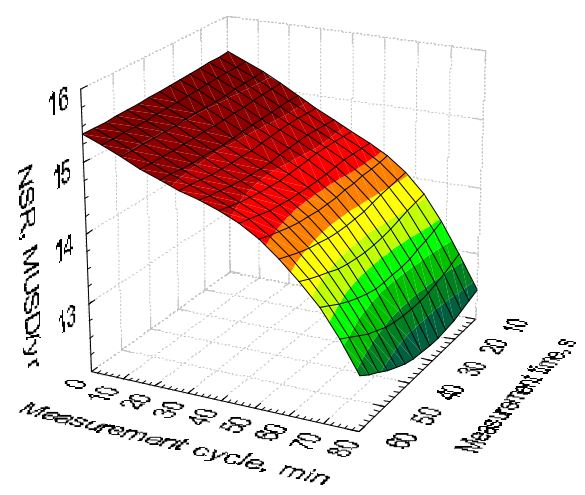

Fig. 8. Smoothed surface of the net smelter return as a function of the analyzer cycle time and sample measurement time. 


\section{CONCLUSION}

In this paper, the economical performance of the flotation process as a function of measurement accuracy and sampling frequency was studied. A dynamic flotation simulation model was developed for this purpose. Although the model is simple and calculation of the economical performance depends on cross assumptions, the results give some quantification of the significance of analyzer accuracy and precision and speed of the analysis. The economical performance of the flotation circuit decreases drastically if the control actions are delayed enough. To reduce the error due to the measurement delay, fast basic measurement and control are necessary to complement process analyzers, and to keep the process stable before the next assay arrives. Froth image analysis, as presented by Brown et al (2001), can be used for this purpose.

\section{REFERENCES}

Bascur, O.A. (1982), Modelling and computer control of a flotation cell, University of Utah, Salt Lake City, 372 p.

Brown, N.M., Dioses J.,Van Olst M. (2001). Advances in Flotation Process Control at Cadia Hill Gold Mine Using Froth Imaging Technology, 2001 SME Annual Meeting, Denver.

Casali, A., Gonzales, G., Agusto, H., Vallebuona, G. (2002), Dynamic Simulator of a rougher flotation circuit for a copper sulphide ore, Minerals Engineering, 15, 253-262.

Cooper, H.R. (1976). On-Stream X-Ray Analysis in Flotation, AIME, New York. p. 865.

Flintoff, B.C. (1992). Measurement Issues in Quality Control, Proceedings of the 1992 Toronto CMP Branch Meeting, Toronto.

Jämsä-Jounela, S-L.(1990), Modern Approaches to Control of Mineral Processing, Acta Polytecnica Scandinavica, Mathematics and Computer Science Series, 57, Helsinki, 33 p.

Kongas, M., Saloheimo, K., When is the XRF assay good enauogh for process control, Preprint No. 01-189 of SME Annual
Meeting Denver, Colorado - March 26-28, Denver 2001, 5 p.

Lynch, A.J., Johnson N.W, Manlapig, E.V., Thorne, C.G. (1981), Developments in Mineral Processing 3, Mineral and Coal Flotation Circuits, Their Simulation and Control, Elsevier, Amsterdam, 290 p.

Lähteenmäki S, Miettunen J, Saloheimo K (1999). 30 years of on-stream analysis at the Pyhäsalmi mine, Preprints of the 1999 SME Annual Meeting, Denver.

Tenno, R. and Jämsä-Jounela, S-L. (1996), Copper Flotation Profit and Control System Accuracy, Control Engineering Practice, Vol. 4, No. 11, p. 1545-1551. 